\section{Testing for antineutrophil cytoplasmic antibodies (ANCAs) in patients with systemic vasculitides and other diseases}

To the editor,

In the excellent study recently published in the Annals of the Rheumatic Disease, ${ }^{1}$ Damoiseaux et al showed a high diagnostic performance of antigen-specific immunoassay for the detection of myeloperoxidase (MPO) and proteinase 3 (PR3) antineutrophil cytoplasmic antibodies (ANCAs). These data challenge the role of indirect immunofluorescence in the ANCA testing algorithm. In our centre, we have discarded ANCA indirect immunofluorescence more than a decade ago. Therefore, new data showing the feasibility of screening by antigen-specific immunoassay have a particular value for us. In the recent series of 284 patients with ANCA-associated vasculitides, we have detected ANCAs by this approach in $96.9 \%$ of patients with microscopic polyangiitis (MPA) but only in $72.7 \%$ of patients with granulomatosis with polyangiitis (GPA) (table 1). The latter result can be explained by a relatively high occurrence of localised GPA in our series, ${ }^{2}$ since a rate of ANCA positivity reached $92.2 \%$ in patients with renal GPA.

ANCA testing should be performed only in the clinical context since PR3-ANCA and MPO-ANCA can be found in the other conditions than vasculitis, for example, infective endocarditis, ${ }^{3}$ tuberculosis, ${ }^{4}$ primary sclerosing cholangitis ${ }^{5}$ and interstitial lung diseases. ${ }^{6}$ The results of several studies suggest that in such patients, ANCAs have not been merely a chance finding and may be clinically relevant, for example, a high prevalence of ANCAs was identified in unselected patients with infective endocarditis (24\%). Seropositive patients presented more commonly with a subacute form of infective endocarditis leading to multiple valve involvement and a more frequent renal impairment. ${ }^{3}$ Recent evidence indicates that a proportion of patients with idiopathic pulmonary fibrosis who were MPO-ANCA positive at diagnosis or who subsequently seroconverted can develop MPA. ${ }^{7}$ The incidence of MPA tended to be lower in patients treated than not treated with corticosteroids though the difference did not reach statistical significance. In the other study, PR3-ANCAs were detected in a significant proportion of patients with primary sclerosing cholangitis compared with other liver diseases including primary biliary cirrhosis and autoimmune hepatitis. PR3-ANCAs were not solely related to underlying inflammatory bowel disease and may be a specific biomarker for primary sclerosing cholangitis. ${ }^{5}$

Damoiseaux et al suggested that ANCA-associated vasculitides may be classified based on the ANCA serotype since recent studies have shown that PR3-ANCA and MPO-ANCA diseases are strongly associated with distinguishable genetic alleles,

\begin{tabular}{|c|c|c|c|}
\hline & All patients $(n=284)$ & GPA $(n=220)$ & MPA $(n=64)$ \\
\hline PR-ANCA & $145(51.1)$ & $127(57.7)$ & $18(28.1)$ \\
\hline MPO-ANCA & $63(22.2)$ & $27(12.2)$ & $36(56.3)$ \\
\hline Both types & $9(3.2)$ & $5(2.2)$ & $4(6.2)$ \\
\hline Undifferentiated & $5(1.8)$ & $1(0.5)$ & $4(6.2)$ \\
\hline Negative & $62(21.8)$ & $60(27.3)$ & $2(3.1)$ \\
\hline
\end{tabular}

ANCA, antineutrophil cytoplasmic antibody; GPA, granulomatosis with polyangiitis; $\mathrm{MPA}$, microscopic polyangiitis; MPO, myeloperoxidase; $\mathrm{PR}$, proteinase. phenotypic differences and differences in risk of relapse and response to immunosuppressive treatment. However, not all studies confirm a predictive value of ANCA specificity in patients with ANCA-associated vasculitis. Miloslavsky $e t a l^{8}$ in a pooled analysis of the Wegener's Granulomatosis Etanercept Trial and the Rituximab in Associated Vasculitis (ANCA) (RAVE) trial were unable to demonstrate the important clinical differences between patients who were MPO-ANCA positive and PR3-ANCA positive and with GPA. A relapse rate in patients who were MPO-ANCA positive and with GPA was higher than in patients who were MPO-ANCA positive and with MPA at 12 and 18 months. Therefore, in this patient cohort, a risk of relapse was associated more closely with the disease type than with ANCA specificity.

GPA and MPA have many overlapping features, and nosological diagnosis per se usually does not determine a choice of treatment. ${ }^{9}$ Nevertheless, patients with GPA frequently present with extravascular granulomatous lesions (orbital pseudotumour, necrotising rhinitis and persisting lung infiltrates) that are not seen in MPA. Predominant granulomatous lesions may have impact on the choice of immunosuppression, for example, rituximab may be less effective for the induction of remission in such patients. ${ }^{10}$ Up to $15 \%-25 \%$ of patients with GPA present with the localised form of disease that is restricted to the upper respiratory tract, eyes and ears. These patients have better survival and require less aggressive remission induction treatment compared with that in renal or other organ-threatening disease. ${ }^{9}$ They usually show predominant granulomatous lesions and, therefore, may be less responsive to rituximab. Moreover, ANCA negativity is more prevalent in patients with the localised GPA.

The ANCA specificity-based classification will apparently be more user friendly than a nosological scheme, but will it improve treatment? ANCA specificity may predict a risk of relapse but its predictive value for outcomes, such as end-stage renal disease or death is low, if any. Patients who are PR3ANCA positive may require longer maintenance treatment, for example, at least 36 months as opposed to 24 months in patients who are MPO-ANCA positive. However, according to the latest European League Against Rheumatism/European Renal Association - European Dialysis and Transplant Association (ERA-EDTA) recommendations for the management of ANCAassociated vasculitis, a choice of initial remission-induction treatment depends on the presence of organ or life-threatening disease. $^{9}$ ANCA specificity was not incorporated in these recommendations. Moreover, it was stated that structured clinical assessment rather than ANCA testing should inform decisions on changes in treatment for ANCA-associated vasculitis. This statement was added in the context of serial ANCA testing as a means of predicting future relapse. However, it seems to be relevant for the treatment choices in general.

ANCA presence and specificity not only aids diagnosis of ANCA-associated vasculitis but also may have important value as a guide for immunosuppressive treatment. Nevertheless, different histological and clinical features of ANCA-associated vasculitis are more relevant for treatment decisions than any laboratory parameter (predominant granulomatous lesions, localised vs generalised disease, renal vs non-renal vasculitis, relapsing vs non-relapsing disease, etc).

In conclusion, Damoiseaux et al data warranting a revision of the international consensus on screening for ANCA are of significant value for rheumatologists caring for patients GPA and MPA. 
Pavel Novikov, ${ }^{1}$ llya Smitienko, ${ }^{2}$ Nikolay Bulanov, ${ }^{1}$ Anastasiia Zykova, ${ }^{3}$ Sergey Moiseev ${ }^{1,3}$

${ }^{1}$ Clinic of Nephrology, Internal and Occupational Diseases, Sechenov First Moscow State Medical University, Moscow, Russia

${ }^{2}$ Russian University of Peoples' Friendship, Moscow, Russia

${ }^{3}$ Faculty of Medicine, Lomonosov Moscow State University, Moscow, Russia

Correspondence to Professor Sergey Moiseev, Clinic of Nephrology, Internal and Occupational Diseases, Sechenov First Moscow State Medical University, Rossolimo 11/5, Moscow 119435, Russia; clinpharm@mtu-net.ru

Contributors All authors contributed to review of the manuscript.

Competing interests None declared.

Provenance and peer review Not commissioned; internally peer reviewed.

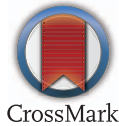

To cite Novikov P, Smitienko I, Bulanov N, et al. Ann Rheum Dis 2017;76:e23.

Received 28 November 2016

Accepted 1 December 2016

Published Online First 2 January 2017

Ann Rheum Dis 2017;76:e23. doi:10.1136/annrheumdis-2016-210890

\section{REFERENCES}

1 Damoiseaux J, Csernok E, Rasmussen N, et al. Detection of antineutrophil cytoplasmic antibodies (ANCAs): a multicentre European Vasculitis Study Group
(EUVAS) evaluation of the value of indirect immunofluorescence (IIF) versus antigen-specific immunoassays. Ann Rheum Dis 2017;76:647-53.

2 Novikov PI, Moiseev SV, Kuznetsova El, et al. Changing patterns of clinical severity and risk of mortality in granulomatosis with polyangiitis over four decades: the Russian experience. Rheumatol Int 2015;35:891-8.

3 Langlois V, Lesourd A, Girszyn N, et al. Antineutrophil cytoplasmic antibodies associated with infective endocarditis. Medicine (Baltimore) 2016;95: e2564.

4 Esquivel-Valerio JA, Flores-Suárez LF, Rodríguez-Amado J, et al. Antineutrophil cytoplasm autoantibodies in patients with tuberculosis are directed against bactericidal/permeability increasing protein and are detected after treatment initiation. Clin Exp Rheumatol 2010;28(Suppl 57): 35-9.

5 Stinton LM, Bentow C, Mahler M, et al. PR3-ANCA: a promising biomarker in primary sclerosing cholangitis (PSC). PLOS ONE 2014;9:e112877.

6 Hozumi H, Enomoto N, Oyama Y, et al. Clinical implication of proteinase-3-antineutrophil cytoplasmic antibody in patients with idiopathic interstitial pneumonias. Lung 2016;194:235-42.

7 Kagiyama N, Takayanagi N, Kanauchi T, et al. Antineutrophil cytoplasmic antibody-positive conversion and microscopic polyangiitis development in patients with idiopathic pulmonary fibrosis. BMJ Open Respir Res 2015;2: e000058.

8 Miloslavsky EM, Lu N, Unizony S, et al. Myeloperoxidase-ANCA-positive and ANCA-negative patients with granulomatosis with polyangiitis: distinct patient subsets. Arthritis Rheumatol 2016;68:2945-52.

9 Yates M, Watts RA, Bajema IM, et al. EULAR/ERA-EDTA recommendations for the management of ANCA-associated vasculitis. Ann Rheum Dis 2016:75:1583-94.

10 Charles P, Bienvenu B, Bonnotte B, et al. Rituximab: recommendations of the French Vasculitis Study Group (FVSG) for induction and maintenance treatments of adult, antineutrophil cytoplasm antibody-associated necrotizing vasculitides. Presse Med 2013:42:1317-30 